\title{
Analytical study of higher order modes of elliptical cavities using oblate spheroidal eigenvalue solution
}

\author{
V. Jain, ${ }^{1}$ U. V. Bhandarkar, ${ }^{2}$ S. C. Joshi, ${ }^{1}$ and S. Krishnagopal ${ }^{3,4, *}$ \\ ${ }^{1}$ PLSCD, Raja Ramanna Centre for Advanced Technology, Indore 452013, India \\ ${ }^{2}$ Department of Mechanical Engineering, Indian Institute of Technology Bombay, Mumbai 400076, India \\ ${ }^{3}$ Bhabha Atomic Research Centre, Mumbai 400085, India \\ ${ }^{4}$ Centre for Excellence in Basic Sciences, Mumbai 400098, India
}

(Received 6 January 2011; published 14 April 2011)

\begin{abstract}
The oblate spheroidal shape is close to the commonly used elliptical rf cavity shape employed in accelerators. Here we solve the oblate spheroidal radial and angular wave functions to obtain the frequencies of the axisymmetric TM and TE modes. We develop a semianalytic formalism to calculate the characteristic parameters, such as shunt impedance, of higher order modes (HOMs). Our formulation is applied to calculate the HOM frequencies of the INDUS-2 and ILC cavities, and the agreement with three-dimensional finite element calculations is excellent. Using this formalism we investigate the effect of changing the oblate shape, and predict an optimized range of $\xi_{0}$ (one of the key parameters to define the geometry), to reduce the number of significant HOMs.
\end{abstract}

DOI: $10.1103 /$ PhysRevSTAB.14.042002

PACS numbers: $29.20 .-\mathrm{c}, 85.25 .-\mathrm{j}, 84.40 .-\mathrm{x}$

\section{INTRODUCTION}

The solution for the eigenfrequencies of any rf cavity depends on its boundary shape. Available analytical calculations are limited to regular shapes such as the rectangular box, the cylinder (pillbox), and the sphere where the Helmholtz and Laplace equations are solved in the respective (Cartesian, cylindrical, and spherical) coordinates to obtain the eigenfrequencies [1]. However, these standard shapes are not among any popular accelerating cavity structures. In fact, the cavity shape is determined by the particle energy and the frequency of the rf cavity. Besides these, other parameters such as shunt impedance, quality factor, surface electric and magnetic fields, and HOMs also affect the overall cavity shape and dimensions. Based on some of these constraints, the elliptically shaped $\pi$-mode cavity is a general choice for accelerators [2]. In $\pi$-mode structures the cavity gap is $\beta \lambda / 2$, where $\beta$ is the ratio of particle velocity to the velocity of light and $\lambda$ is the wavelength of the cavity resonating frequency. Such elliptical shaped cavities resemble the spheroidal oblate shape, where it is possible to obtain the analytical solution by solving the wave equation in spheroidal coordinates. Hence, the study of oblate spheroidal shapes is useful from the point of view of accelerating cavities.

The design of any standard axisymmetric rf cavity shape is generally defined by eight independent parameters: cavity gap $\left(C_{g}\right)$, cavity radius $\left(C_{r}\right)$, cavity beam tube radius

\footnotetext{
*skrishna@tifr.res.in

Published by American Physical Society under the terms of the Creative Commons Attribution 3.0 License. Further distribution of this work must maintain attribution to the author(s) and the published article's title, journal citation, and DOI.
}

$\left(C_{b}\right)$, wall angle $(\alpha)$, iris ellipse dimensions $\left(a_{i}, b_{i}\right)$, and equator ellipse dimensions $\left(a_{e}, b_{e}\right)$. For many designs, the wall angle is positive, and in this case, the cavity shape resembles the spheroidal oblate shape-except near the axis (see Fig. 1) - where it is possible to obtain the analytical solution by solving the wave equation in spheroidal coordinates. Hence, the study of oblate spheroidal shapes is useful from the point of view of studying axisymmetric modes in elliptical cavities.

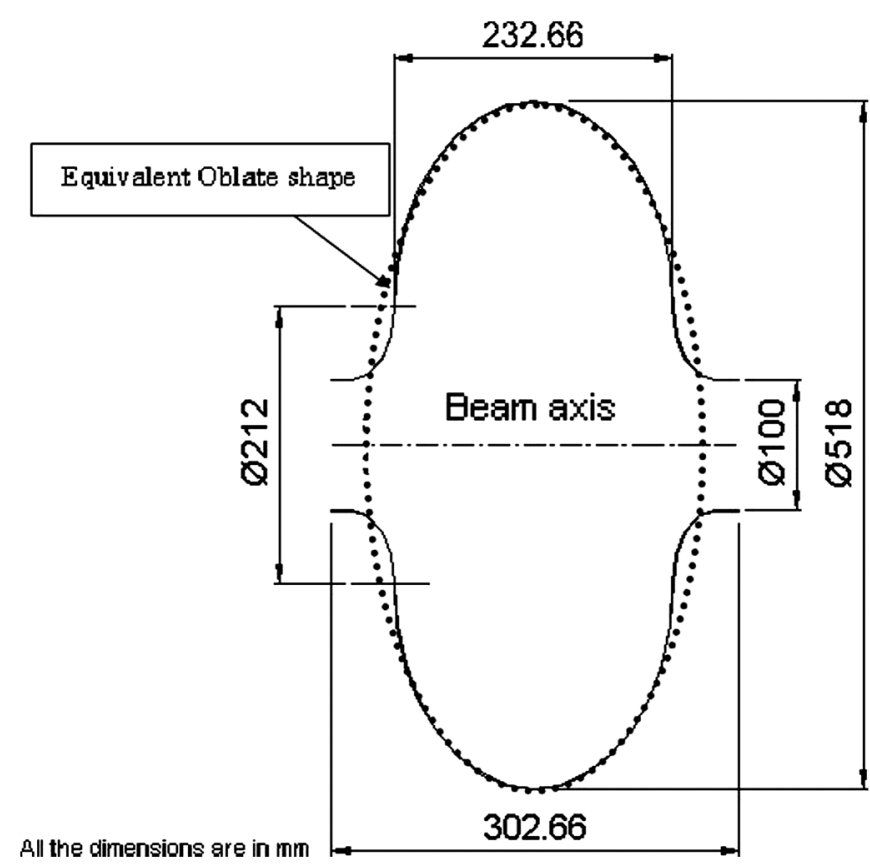

FIG. 1. Profile of a typical elliptical rf cavity (INDUS-2 rf cavity in bold line) and equivalent oblate shape (dashed line). 
The work on analytical formulations for spheroidal eigenfrequency has been carried out by many researchers. Kokkorakis and Roumeliotis $[3,4]$ have tabulated the coefficients of a series solution of spheroidal eigenfrequencies. However, their solution was valid only for small perturbations from the spherical shape. Li et al. [5,6] similarly obtained eigenfrequencies for a spheroidal conducting cavity with better accuracy and for a slightly higher deviation from the spherical shape. However, both of these are not applicable to realistic oblate shaped cavities used in accelerators.

While obtaining the eigenfrequencies, the accuracy of the solutions depends upon the calculation of spheroidal angular and radial wave functions. The formulation of these harmonics in terms of series solutions has been well documented by Stratton et al. earlier in 1956 [7] and Flammer in 1957 [8]. Apart from this, these harmonics are tabulated in terms of a power series expansion by Abramowitz and Stegun [9]. Li et al. [10] have reviewed various methodologies for evaluating the spheroidal angular and radial functions (for both prolate and oblate shapes) and their eigenvalues. Thompson [11,12] has also solved the spheroidal wave harmonics by using rigorous computations. Zhang and Jin [13] have provided FORTRAN programs for accurate calculation of these functions. However, these solutions have been used for applications such as wave propagation using antennas $[5,14]$ and fluid dynamics [15], and have not yet been used to obtain the eigenfrequencies of oblate spheroidal shapes with large eccentricity.

In the present work, the eigenfrequencies for general oblate shapes are obtained with high accuracy for nearly the entire range of eccentricities. A least-squares polynomial fit has been formulated which provides a ready solution of the eigenfrequencies of oblate shapes. Further, the radial and angular functions have also been fitted with a polynomial to obtain the characteristics constants of the fundamental and higher order axisymmetric modes of $\mathrm{rf}$ cavities. The cavity frequencies and characteristic constants are verified by comparing these values with those of the INDUS-2 rf cavity [16] and the ILC cavity [17]. Finally, the effect of variation in the cavity shapes is studied in detail in an effort to obtain cavities with reduced effectiveness of HOMs.

It should be noted that, as with any analytic work, there are limitations in applying the results to actual, operating, cavities. First, the analytic shape is symmetric and explicitly excludes modes with nonzero azimuthal index. Second, for cavity shapes that have nose cones, such as the Cornell cavity, the deviation from the oblate shape is large, and our analysis is not applicable. Third, since the deviations from the oblate spheroidal shape are greatest at the iris, this approach is not useful for calculating the peak electric field, which is defined by the iris area. Finally, penetrations, such as the beam pipe, will perturb the geometry, and change the modes. The last issue has been addressed by comparing our analytic results with those of detailed finite element method (FEM) simulations of an actual cavity geometry, and with experiment. We find that deviations in the higher-order mode frequencies are within $5 \%-10 \%$, which gives us confidence in the usefulness of our analytic approach.

\section{SPHEROIDAL COORDINATES AND CORRESPONDING WAVE EQUATION}

There are two types of spheroids: prolate and oblate. A prolate spheroid is formed by rotating an ellipse around its major axis while an oblate spheroid is formed by rotating an ellipse around its minor axis. Since typical accelerating cavities in accelerators are close to the oblate geometry, we focus only on oblate spheroids. Figure 2 shows the geometry of the oblate spheroid, which can be described by the curvilinear orthogonal coordinate system $(\eta, \xi, \phi)$. These coordinates vary in the range $-1<\eta<1,0<\xi<\infty$, and $0<\phi<2 \pi$ for oblate cavities [7-13].

They are related to the Cartesian coordinates $(x, y, z)$ by

$$
\begin{gathered}
x=\frac{d}{2} \cos \phi \sqrt{\left(1-\eta^{2}\right)\left(\xi^{2}+1\right)} \\
y=\frac{d}{2} \sin \phi \sqrt{\left(1-\eta^{2}\right)\left(\xi^{2}+1\right)} \\
z=\frac{d}{2} \eta \xi .
\end{gathered}
$$

In the above equations $d$ is the distance between the two foci, and is given by $d=\sqrt{a^{2}-b^{2}}$, where $a$ is the length

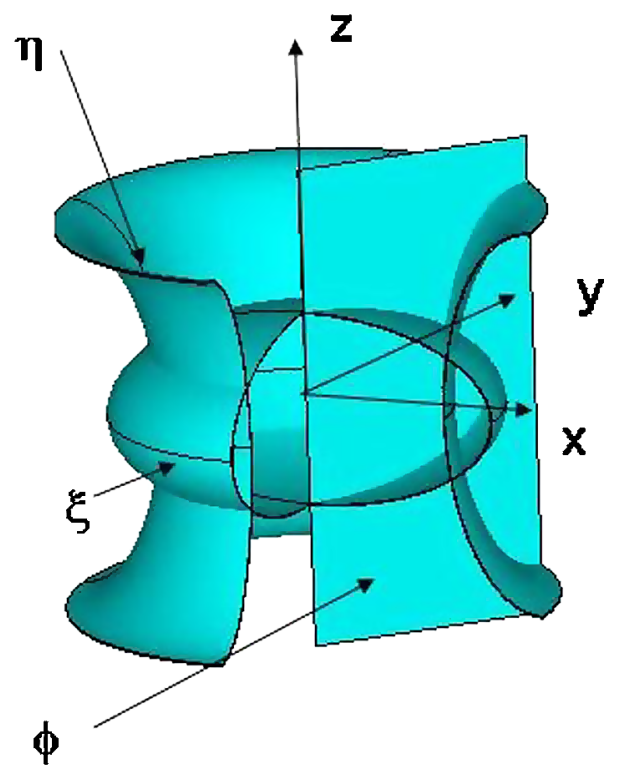

FIG. 2. Oblate spheroidal coordinates $\eta, \xi$, and $\phi$ for constant parameter values. 
of the major axis and $b$ is the length of the minor axis of the ellipse.

Ellipses of various sizes are described in the oblate spheroidal system by the parameter $\xi$, which is the ratio of the axis of rotation to the focal distance of the ellipse. For the oblate system, $\xi \geq 0$, with $\xi=0$ representing a disk and $\xi \rightarrow \infty$ representing a sphere. Figure 3 shows the variation of $\xi$ with the ellipse ratio (ratio of minor axis to major axis). The parameter $\eta$ describes a system of hyperbolas and the parameter $\phi$ describes a plane and is identical to the variable $\phi$ in the spherical coordinate system.

The oblate spheroidal coordinates form a curvilinear orthogonal system, with metric coefficients given by

$$
\begin{aligned}
& h_{\eta}=\frac{d}{2} \sqrt{\frac{\xi^{2}+\eta^{2}}{1-\eta^{2}}}, \\
& h_{\xi}=\frac{d}{2} \sqrt{\frac{\xi^{2}+\eta^{2}}{\xi^{2}-1}}, \\
& h_{\phi}=\frac{d}{2} \sqrt{\left(1-\eta^{2}\right)\left(\xi^{2}+1\right)} .
\end{aligned}
$$

The Helmholtz wave equation in these coordinates can be written as

$$
\begin{aligned}
& \frac{\partial}{\partial \eta}\left(\frac{h_{\xi} h_{\phi}}{h_{\eta}} \frac{\partial W}{\partial \eta}\right)+\frac{\partial}{\partial \xi}\left(\frac{h_{\eta} h_{\phi}}{h_{\xi}} \frac{\partial W}{\partial \xi}\right)+\frac{\partial}{\partial \phi}\left(\frac{h_{\xi} h_{\eta}}{h_{\phi}} \frac{\partial W}{\partial \phi}\right) \\
& \quad+k^{2} h_{\eta} h_{\xi} h_{\phi} W=0,
\end{aligned}
$$

where $W$ is the wave function and $k(=2 \pi / \lambda)$ is the wave number.

For an oblate system, substituting for the metric coefficients from Eq. (4) in the wave equation [11-13], one obtains

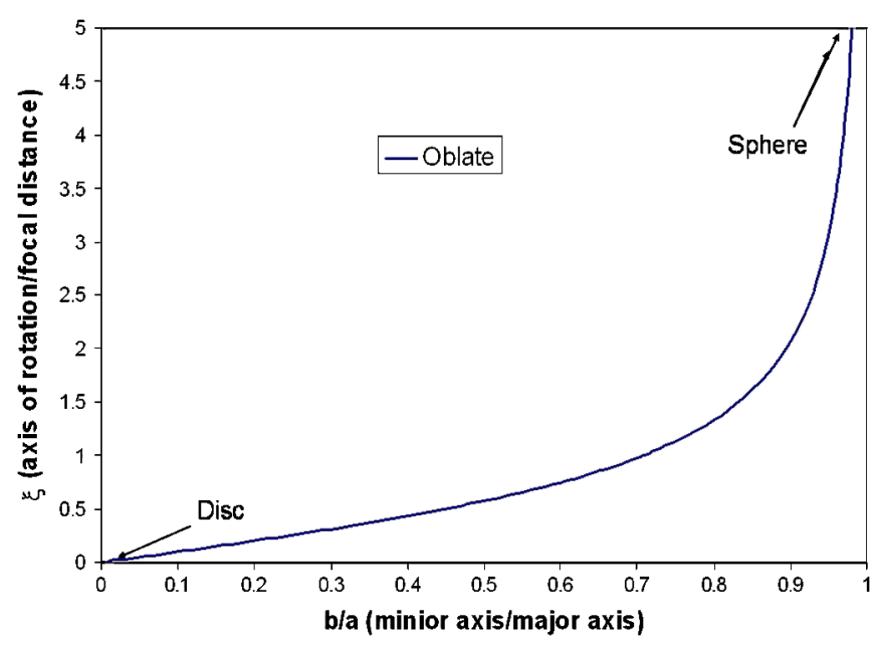

FIG. 3. Plot of $\xi$ as a function of the ratio of minor axis to major axis.

$$
\begin{aligned}
& \frac{\partial}{\partial \eta}\left(\left(1-\eta^{2}\right) \frac{\partial W}{\partial \eta}\right)+\frac{\partial}{\partial \xi}\left(\left(\xi^{2}+1\right) \frac{\partial W}{\partial \xi}\right) \\
& +\frac{\eta^{2}+\xi^{2}}{\left(1-\eta^{2}\right)\left(\xi^{2}+1\right)} \frac{\partial^{2} W}{\partial \phi^{2}}+c^{2}\left(\xi^{2}+\eta^{2}\right) W=0
\end{aligned}
$$

where $c=k d / 2=\pi d / \lambda$.

This equation can be solved by separation of variables as follows. Write

$$
W(\eta, \xi, \phi, c)=S_{m n}(-i c, \eta) R_{m n}(-i c, i \xi) \Phi(\phi),
$$

where $S_{m n}(-i c, \eta)$ and $R_{m n}(-i c, i \xi)$ are the spheroidal angular and radial functions, respectively. Here $c$ is included explicitly to indicate that it is a parameter in both $S$ and $R$. Substituting Eq. (7) in Eq. (6) and separating variables, one obtains differential equations for the angular $S_{m n}(-i c, \eta)$ and the radial function $R_{m n}(-i c, i \xi)$ as

$$
\begin{aligned}
& \frac{d}{d \eta}\left(\left(1-\eta^{2}\right) \frac{d S_{m n}(-i c, \eta)}{d \eta}\right) \\
& \quad+\left(\lambda_{m n}+c^{2} \eta^{2}-\frac{m^{2}}{1-\eta^{2}}\right) S_{m n}(-i c, \eta)=0, \\
& \frac{d}{d \xi}\left(\left(\xi^{2}+1\right) \frac{d R_{m n}(-i c, i \xi)}{d \xi}\right) \\
& \quad-\left(\lambda_{m n}-c^{2} \xi^{2}-\frac{m^{2}}{\xi^{2}+1}\right) R_{m n}(-i c, i \xi)=0 .
\end{aligned}
$$

Here $m$ and $n$ are separation constants and $\lambda_{m n}$ is referred to as the characteristic value.

As mentioned earlier when $\xi \rightarrow \infty$ (i.e. $d \rightarrow 0$, hence $c \rightarrow 0$ ), the spheroid becomes a sphere. Accordingly, the spheroidal angular wave function $S_{m n}(-i c, \eta)$ becomes the associated Legendre function, and the spheroidal radial wave function $R_{m n}(-i c, i \xi)$ becomes the spherical Bessel function. Therefore, the spheroidal angular wave function $S_{m n}(-i c, \eta)$ can be expanded in an infinite series of the associated Legendre functions, and the spheroidal radial wave function $R_{m n}(-i c, i \xi)$ can be expanded in an infinite series of the spherical Bessel functions [7-13].

The spheroidal angular functions $S_{m n}(-i c, \eta)$ are usually expanded into spherical Legendre functions of the first kind, $P_{m+k}^{m}(\eta)$, or the second kind, $Q_{m+k}^{m}(\eta)$. For functions of the first kind and the second kind, which are regular at $n= \pm 1$, one can write [13]

$$
S_{m n}^{1}(-i c, \eta)=\sum_{k=0,1}^{\infty} d_{k}^{m n}(-i c) P_{m+k}^{m}(\eta)
$$

and

$$
S_{m n}^{2}(-i c, \eta)=\sum_{k=0,1}^{\infty} d_{k}^{m n}(-i c) Q_{m+k}^{m}(\eta),
$$

where the superscripts 1 and 2 refer to the first and second kind, respectively, and $d_{k}^{m n}(-i c)$ are the expansion coefficients which are determined by solving a three-term 
recurrence relation [13] obtained by substituting Eqs. (10) and (11) into Eq. (8) (for both first and second kinds). As an example, the plot of the angular wave function of the first kind and its derivative for $m=1, n=1$, and $c=1.0$ is shown in Fig. 4. The spheroidal angular wave functions are calculated using a FORTRAN program.

The spheroidal radial functions, $R_{m n}(-i c, i \xi)$, are usually expanded on the basis of spherical Bessel, Neumann, or Hankel functions and can be written as [13]

$$
\begin{aligned}
R_{m n}^{l}(-i c, i \xi)= & {\left[\sum_{k=0,1}^{\infty} \frac{(2 m+k) !}{k !} d_{k}^{m n}(-i c)\right]^{-1}\left(1-\frac{1}{\xi^{2}}\right)^{m / 2} } \\
& \times \sum_{k=0,1}^{\infty} i^{k+m-n} \frac{(2 m+k) !}{k !} d_{k}^{m n}(-i c) Z_{m+k}^{(l)}(c \xi),
\end{aligned}
$$

where $d_{k}^{m n}(-i c)$ are the expansion coefficients defined earlier for the spheroidal angular wave functions. $Z_{m+k}^{(l)}(c \xi)$ represents the spherical Bessel functions given by

$$
\begin{aligned}
Z^{(l)}(z) & =\sqrt{\frac{\pi}{2 z} J_{n+1 / 2}(z)} \\
& =j_{n}(z)(\text { spherical Bessel function) } \\
Z^{(2)}(z) & =\sqrt{\frac{\pi}{2 z} Y_{n+1 / 2}(z)} \\
& =y_{n}(z) \text { (spherical Neumann function) }
\end{aligned}
$$

$$
\begin{aligned}
Z^{(3)}(z) & =j_{n}(z)+i y_{n}(z) \\
& =h_{n}^{1}(z) \text { (spherical Hankel function of first kind) }
\end{aligned}
$$

$$
\begin{aligned}
Z^{(4)}(z) & =j_{n}(z)-i y_{n}(z) \\
& =h_{n}^{2}(z)(\text { spherical Hankel function of second kind }) .
\end{aligned}
$$

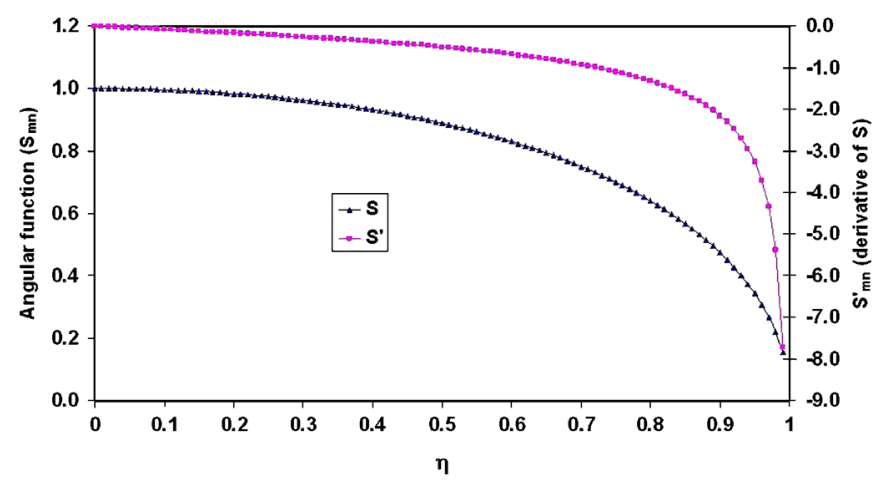

FIG. 4. Angular function of first kind $S_{m n}$ and its derivative $S_{m n}^{\prime}$ for $m=1, n=1$, and $c=1.0$.

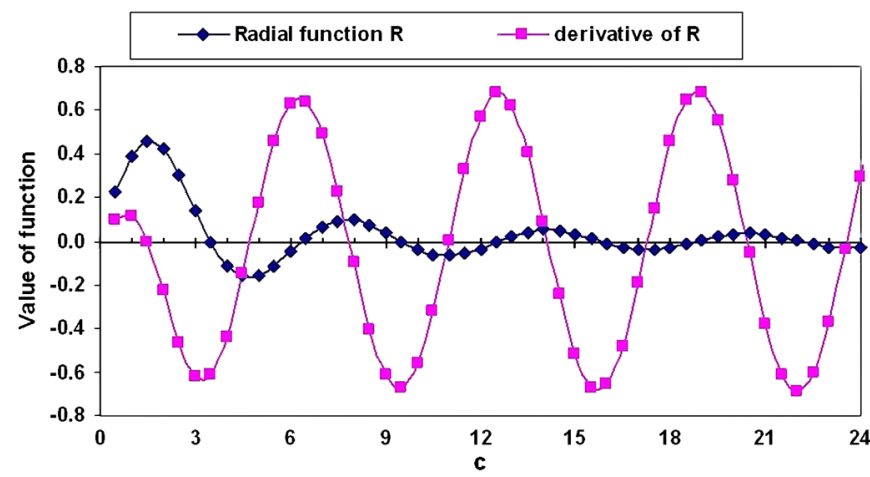

FIG. 5. Radial function and its derivative of first kind for $m=$ $1, n=1$, and $\xi=1$.

The radial function of the first kind and its derivative are plotted in Fig. 5, by varying the value of $c$, for $m=1$, $n=1$, and $\xi=1$. The radial functions are calculated using another FORTRAN program.

\section{CAVITY ELECTRODYNAMICS IN OBLATE SPHEROIDAL COORDINATES}

\section{A. TM AND TE MODE FORMULATIONS}

Assuming a harmonic time dependence of the magnetic (H) and electric (E) fields, the wave equations for the fields can be written in the axisymmetric oblate spheroidal coordinate system, i.e. $(\eta, \xi)$, as

$$
\begin{aligned}
& \frac{\partial}{\partial \xi}\left(\frac{h_{\eta}}{h_{\phi} h_{\xi}} \frac{\partial\left(h_{\phi} H_{\phi}\right)}{\partial \xi}\right)+\frac{\partial}{\partial \eta}\left(\frac{h_{\xi}}{h_{\phi} h_{\eta}} \frac{\partial\left(h_{\phi} H_{\phi}\right)}{\partial \eta}\right) \\
& \quad+\omega^{2} \mu \varepsilon \frac{h_{\xi} h_{\eta}}{h_{\phi}}\left(h_{\phi} H_{\phi}\right)=0
\end{aligned}
$$

and

$$
\begin{aligned}
& \frac{\partial}{\partial \xi}\left(\frac{h_{\eta}}{h_{\phi} h_{\xi}} \frac{\partial\left(h_{\phi} E_{\phi}\right)}{\partial \xi}\right)+\frac{\partial}{\partial \eta}\left(\frac{h_{\xi}}{h_{\phi} h_{\eta}} \frac{\partial\left(h_{\phi} E_{\phi}\right)}{\partial \eta}\right) \\
& \quad+\omega^{2} \mu \varepsilon \frac{h_{\xi} h_{\eta}}{h_{\phi}}\left(h_{\phi} E_{\phi}\right)=0 .
\end{aligned}
$$

Let $h_{\phi} H_{\phi}=A_{n} U(c, \xi) V(c, \eta)$ for TM modes in Eq. (17), where $A_{n}$ is a constant and $U(c, \xi)$ and $V(c, \eta)$ are functions of $R(-i c, i \xi)$ and $S(-i c, \eta)$, respectively. We then obtain the following two differential equations:

$$
\begin{aligned}
& \frac{d}{2}\left(\xi^{2}+1\right) \frac{d^{2} U}{d \xi^{2}}+\left[d^{3} \xi^{2} \omega^{2} \mu \varepsilon-s\right] U=0, \\
& \frac{d}{2}\left(1-\eta^{2}\right) \frac{d^{2} V}{d \eta^{2}}+\left[d^{3} \eta^{2} \omega^{2} \mu \varepsilon-s\right] V=0 .
\end{aligned}
$$

Here $s$ is the separation constant.

Equations (19) and (20) can be compared with the oblate spheroidal Eqs. (8) and (9). For the axisymmetric case 
(and for $m=1$ ), the $U$ and $V$ as given in Refs. [5,6] can be written in terms of $R$ and $S$ as

$$
\begin{aligned}
& U(c, \xi)=\sqrt{\left(\xi^{2}+1\right)} R_{1 n}^{i}(-i c, i \xi), \\
& V(c, \eta)=\sqrt{\left(1-\eta^{2}\right)} S_{1 n}(-i c, \eta)
\end{aligned}
$$

Then, for the TM modes, the fields $H_{\phi}, E_{\xi}$, and $E_{\eta}$ can be written as

$$
\begin{gathered}
H_{\phi}=\frac{A_{n}}{h_{\phi}}\left[\sqrt{\left(\xi^{2}+1\right)} R_{1 n}^{i}(-i c, i \xi)\right]\left[\sqrt{1-\eta^{2}} S_{1 n}(-i c, \eta)\right] \\
E_{\xi}=\frac{1}{j \omega h_{\eta} h_{\phi}} \frac{\partial}{\partial \eta}\left(h_{\phi} H_{\phi}\right) \\
E_{\eta}=\frac{1}{j \omega h_{\xi} h_{\phi}} \frac{\partial}{\partial \xi}\left(h_{\phi} H_{\phi}\right)
\end{gathered}
$$

The surface of an oblate spheroid is defined by $\xi=\xi_{0}$, a constant. For metallic spheroids (conducting boundary) the tangential electric field $E_{\eta}$ is zero at $\xi_{0}$. Hence, from Eq. (25),

$$
\left.\frac{d}{d \xi}\left[\left(\sqrt{\xi^{2}+1}\right) R_{1 n}^{i}(-i c, i \xi)\right]\right|_{\xi=\xi_{0}}=0
$$

By the principle of duality, the field components for the TE modes can be obtained by substituting $E_{\phi}$ for $H_{\phi},-H_{\xi}$ for $E_{\xi}$, and $-H_{\eta}$ for $E_{\eta}$, respectively. Hence, for TE modes $h_{\phi} E_{\phi}=B_{n} U(c, \xi) V(c, \eta)$ (where $B_{n}$ is another constant similar to $A_{n}$ ) and the fields $E_{\phi}, H_{\xi}$, and $H_{\eta}$ can be written as

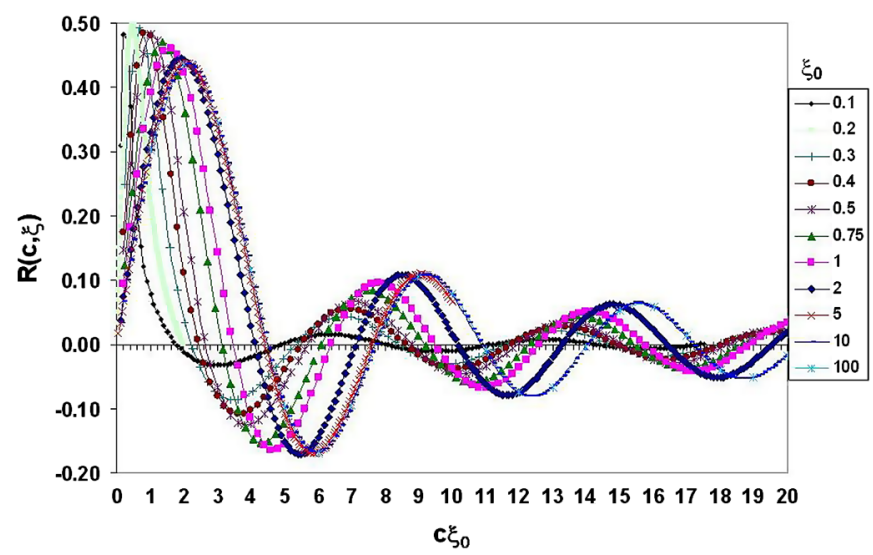

FIG. 6. Function for TE modes plotted against $c \xi$ by varying $\xi_{0}$ from 0.1 to 100 .

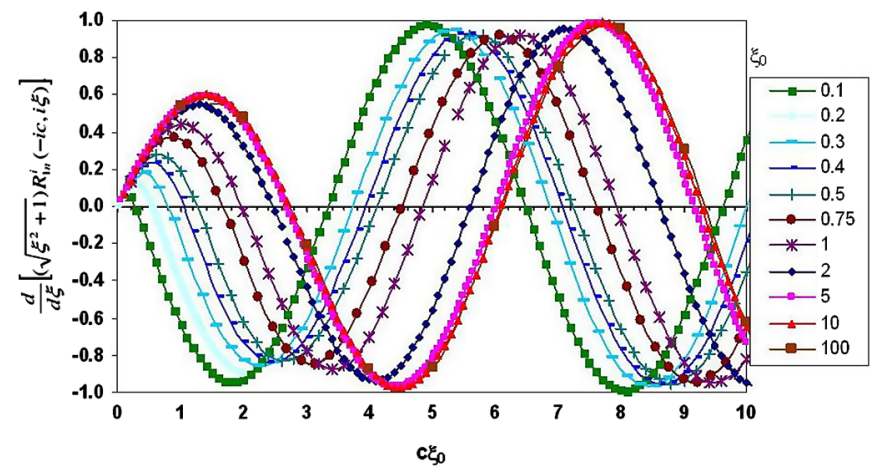

FIG. 7. Function for TM modes plotted against $c \xi_{0}$ by varying $\xi_{0}$ from 0.1 to 100 .

$$
\begin{gathered}
E_{\phi}=\frac{B_{n}}{h_{\phi}}\left[\sqrt{\left(\xi^{2}+1\right)} R_{1 n}^{i}(-i c, i \xi)\right] \\
\times\left[\sqrt{\left(1-\eta^{2}\right)} S_{1 n}(-i c, \eta)\right], \\
H_{\xi}=-\frac{1}{j \omega h_{\eta} h_{\phi}} \frac{\partial}{\partial \eta}\left(h_{\phi} E_{\phi}\right), \\
H_{\eta}=\frac{1}{j \omega h_{\xi} h_{\phi}} \frac{\partial}{\partial \xi}\left(h_{\phi} E_{\phi}\right) .
\end{gathered}
$$

In this case the tangential electric field $E_{\phi}$ goes to zero at $\xi=\xi_{0}$. Hence, the TE modes are given by

$$
\left.\left[R_{1 n}^{i}(-i c, i \xi)\right]\right|_{\xi=\xi_{0}}=0
$$

The frequencies of the TM and TE modes can be calculated by solving Eqs. (26) and (30), i.e., by evaluating the zeros of the radial function and its derivative, for a given $\xi_{0}$, i.e., for a given geometry of the cavity. To study how the mode frequencies vary with cavity geometry, this has to be repeated for different $\xi_{0}$. It is clearly difficult to obtain an analytic expression for the mode frequency as a function of $\xi_{0}$. We have therefore chosen a graphical approach. Figures 6 and 7, respectively, show (for the axisymmetric case, i.e., with $p=0$ ) the plot of the radial function

TABLE I. Mode indices of oblate and pillbox shapes.

\begin{tabular}{cc}
\hline \hline $\begin{array}{c}\text { TM/TE oblate shape } \\
\text { modes }\end{array}$ & $\begin{array}{c}\text { Equivalent TM/TE } \\
\text { pillbox shape modes }\end{array}$ \\
\hline $1,1,0$ & $0,1,0$ \\
$2,1,0$ & $0,1,1$ \\
$3,1,0$ & $0,1,2$ \\
$4,1,0$ & $0,1,3$ \\
$3,2,0$ & $0,2,2$ \\
$2,2,0$ & $0,2,1$ \\
$5,1,0$ & $0,1,4$ \\
$4,2,0$ & $0,2,3$ \\
$6,1,0$ & $0,1,5$ \\
\hline \hline
\end{tabular}




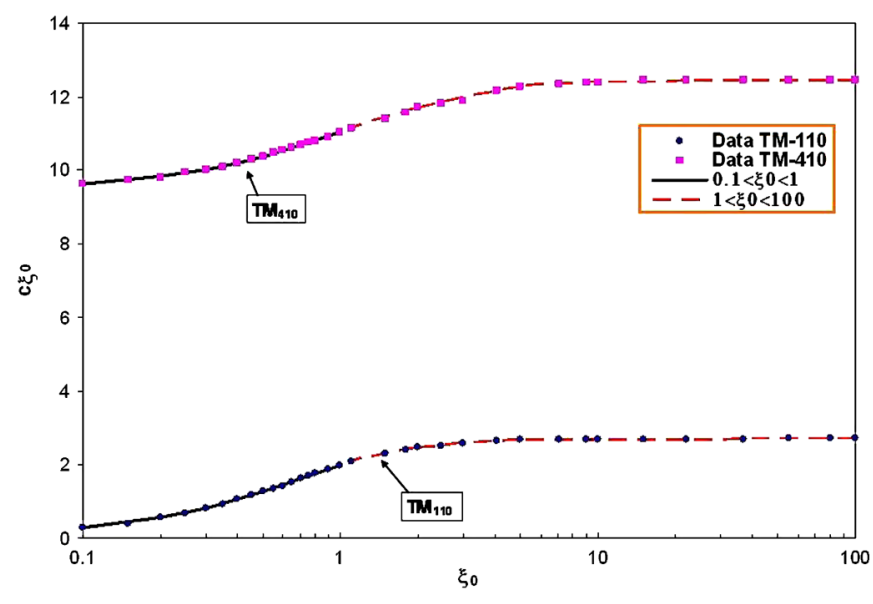

FIG. 8. Frequencies in terms of $c \xi_{0}$ vs $\xi_{0}$ for TM modes.

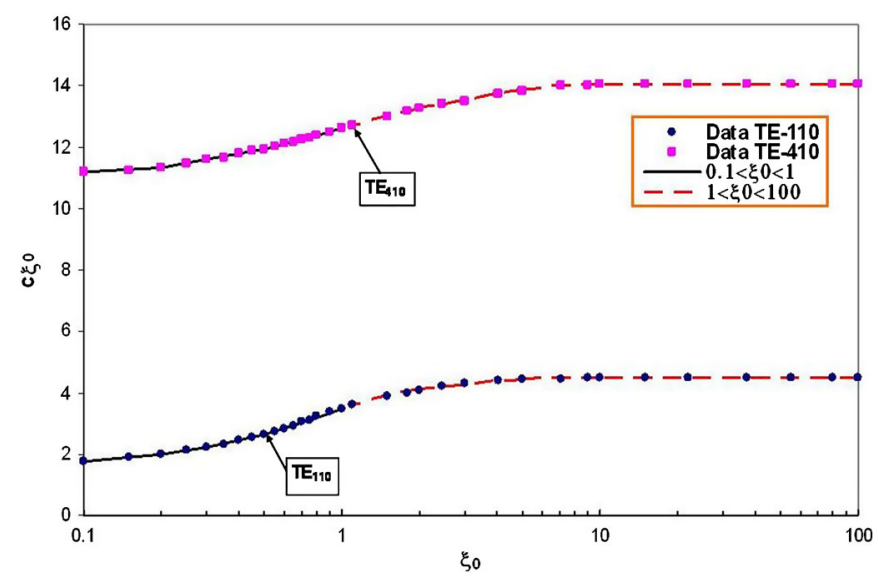

FIG. 9. Frequencies in terms of $c \xi_{0}$ vs $\xi_{0}$ for TE modes.

[Eq. (12)] and its derivative as a function of $c \xi_{0}$, for different $\xi_{0}$ s, ranging from 0.1 to 100 . The variable $c \xi_{0}$ is chosen since the zeros of the function in terms of $c \xi_{0}$ give us the corresponding eigenfrequencies $f_{m n p}$, where the indices $m, n$, and $p$ refer to the periodicities of the fields with reference to $\xi, \eta$, and $\phi$, respectively. For readers used to the nomenclature of the pillbox cavity, it must be mentioned here that the nomenclature of the TM and TE indices for the oblate shape is different from the pillbox (cylindrical) shaped cavities because of the difference in the coordinate system. TM and TE mode indices of the oblate shape that are equivalent to those for the pillbox shape are given in Table I.

For each curve in Figs. 6 and 7, i.e., for each $\xi_{0}$ or each cavity geometry, there will be many zeros, each corresponding to a different mode, the frequency $\left(f_{m n 0}\right)$ of which is determined from the corresponding value of $c$ (since $c=\pi d f_{m n 0} \sqrt{\mu \varepsilon}$ ). For each mode, one can therefore obtain the mode frequency for each $\xi_{0}$, and then plot the mode frequency (or, equivalently, $c \xi_{0}$ ) as a function of $\xi_{0}$, i.e., as a function of changing cavity geometry. We then fit a polynomial expression to this curve to get an analytic expression for mode frequency as a function of $\xi_{0}$. The accuracy of the curve fitting is ensured using a sixth-order fit, and a least-squares algorithm. This is done for each TE and TM mode.

As an example, the exactly calculated frequencies (in terms of $c \xi_{0}$ ) of two TM modes (110 and 410) are plotted as a function of $\xi_{0}$ in Fig. 8 for $\xi_{0}$ ranging from 0.1 to 100 . The corresponding frequencies of the two TE modes (110 and 410) are plotted in Fig. 9. It can be seen that the curves shown in Figs. 8 and 9 have a high degree of nonlinearity in the entire range of $\xi_{0}=0.1$ to 100 . Since a single curve fit would have been poor in this entire range, the range for $\xi_{0}$ is divided into $\xi_{0}=0.1$ to 1 and $\xi_{0}=1$ to 100 for the purpose of fitting. The formulations of this curve fit for the two different ranges are given by polynomial functions in Eqs. (31) and (32), where the coefficients $a_{0}$ to $a_{6}$, and $b_{0}$ to $b_{6}$ are calculated for each TM and TE mode:

$$
\begin{aligned}
f_{m n 0}= & \frac{1}{\pi d \xi \sqrt{\mu \varepsilon}}\left[a_{6} \xi^{6}+a_{5} \xi^{5}+a_{4} \xi^{4}+a_{3} \xi^{3}\right. \\
& \left.+a_{2} \xi^{2}+a_{1} \xi+a_{0}\right] \quad\left(0.1<\xi_{0}<1\right)
\end{aligned}
$$

TABLE II. Coefficients of TM modes for $\xi_{0}=0.1$ to 1 . All the modes have $p=0$.

\begin{tabular}{rccccccrrr}
\hline \hline$m$ & $n$ & $p$ & $a_{6}$ & $a_{5}$ & $a_{4}$ & $a_{3}$ & $a_{2}$ & $a_{1}$ & $a_{0}$ \\
\hline 1 & 1 & 0 & -65.34 & 193.93 & -220.858 & 121.98 & -35.03 & 7.515 & -0.224 \\
1 & 2 & 0 & -0.5 & 0.99 & 0 & -1.23 & 0.48 & 1.969 & 3.143 \\
1 & 3 & 0 & 48.61 & -187.34 & 292.36 & -235.803 & 102.69 & -21.001 & 8.337 \\
2 & 1 & 0 & -33.73 & 133.93 & -212.361 & 169.83 & -71.273 & 16.028 & 0.740 \\
2 & 2 & 0 & -23.59 & 77.56 & -98.49 & 60.13 & -18.272 & 4.424 & 4.609 \\
2 & 3 & 0 & -14.98 & 46.91 & -56.46 & 32.43 & -9.39 & 3.157 & 7.811 \\
3 & 1 & 0 & 0.11 & 0.23 & 0.88 & -3.56 & 0.95 & 5.145 & 0.169 \\
3 & 2 & 0 & -29.7 & 107.13 & -152.968 & 108.89 & -40.89 & 11.739 & 2.599 \\
3 & 3 & 0 & -22.22 & 76.8 & -102.671 & 66.16 & -21.93 & 7.466 & 6.086 \\
4 & 1 & 0 & 73.2 & -233.251 & 293.88 & -185.144 & 58.5 & -4.022 & 1.965 \\
4 & 2 & 0 & -39.68 & 132.967 & -172.054 & 107.08 & -33.44 & 8.833 & 4.480 \\
4 & 3 & 0 & -65.27 & 192.36 & -221.838 & 124.91 & -35.88 & 8.795 & 7.635 \\
\hline \hline
\end{tabular}


TABLE III. Coefficients of TE modes for $\xi_{0}=0.1$ to 1 . All the modes have $p=0$.

\begin{tabular}{rrrrrrrrrr}
\hline \hline$m$ & $n$ & $p$ & \multicolumn{1}{c}{$a_{6}$} & \multicolumn{1}{c}{$a_{5}$} & \multicolumn{1}{c}{$a_{4}$} & \multicolumn{1}{c}{$a_{3}$} & \multicolumn{1}{c}{$a_{2}$} & $a_{1}$ & $a_{0}$ \\
\hline 1 & 1 & 0 & 16.150 & -47.080 & 53.393 & -30.888 & 9.471 & 0.800 & 1.629 \\
1 & 2 & 0 & 0.000 & -0.019 & 0.230 & -0.594 & -0.025 & 2.090 & 4.706 \\
1 & 3 & 0 & 0.000 & -0.016 & 0.200 & -0.508 & -0.111 & 2.075 & 7.849 \\
2 & 1 & 0 & -6.944 & 22.917 & -28.980 & 17.249 & -5.341 & 2.856 & 3.093 \\
2 & 2 & 0 & 4.167 & -15.673 & 23.606 & -18.140 & 6.794 & 0.821 & 6.356 \\
2 & 3 & 0 & -2.083 & 7.901 & -11.579 & 8.081 & -3.231 & 2.562 & 9.399 \\
3 & 1 & 0 & 11.806 & -41.522 & 59.458 & -45.202 & 17.664 & 1.594 & 1.704 \\
3 & 2 & 0 & -9.028 & 22.099 & -14.661 & -3.782 & 6.842 & 1.792 & 4.957 \\
3 & 3 & 0 & 17.361 & -58.574 & 77.257 & -50.692 & 16.225 & 1.667 & 7.975 \\
4 & 1 & 0 & -3.965 & 15.477 & -22.146 & 13.498 & -4.168 & 4.892 & 3.091 \\
4 & 2 & 0 & -0.774 & 4.440 & -7.761 & 5.419 & -2.542 & 4.686 & 6.232 \\
4 & 3 & 0 & 1.679 & -4.571 & 4.796 & -2.877 & 0.131 & 4.201 & 9.401 \\
\hline \hline
\end{tabular}

$$
\begin{aligned}
f_{m n 0} & =\frac{1}{\pi d \xi \sqrt{\mu \varepsilon}}\left[\frac{b_{6}}{\xi^{6}}+\frac{b_{5}}{\xi^{5}}+\frac{b_{4}}{\xi^{4}}+\frac{b_{3}}{\xi^{3}}+\frac{b_{2}}{\xi^{2}}+\frac{b_{1}}{\xi}+b_{0}\right] \\
(1 & \left.<\xi_{0}<100\right) .
\end{aligned}
$$

The coefficients $a_{6}$ to $a_{0}$ of Eq. (31), determined from the least-squares fit, are tabulated in Tables II and III for TM and TE modes, respectively, for the range $\xi_{0}=0.1$ to 1 . Similarly, the coefficients $\left(b_{6}\right.$ to $\left.b_{0}\right)$ can be determined for the range $\xi_{0}=1$ to 100 in Eq. (32). These fits are also shown in Figs. 8 and 9, and it can be seen that the agreement is excellent.

It should be noted that oblate cavities corresponding to $\xi_{0}>1$ are not of practical interest as far as rf accelerating cavities are concerned. For example, the cavities of Tesla $(1.3 \mathrm{GHz})$ and INDUS-2 (505.8 MHz) accelerators have equivalent $\xi_{0}$ values of 0.673 and 0.51 , respectively. Therefore, in the rest of the discussion we focus on the fit given by Eq. (31), using the coefficients from Tables II and III, valid for $\xi_{0}<1$.

\section{B. APPLICATION TO INDUS-2 AND ILC CAVITY HOMS}

In the earlier subsection we have derived a method of predicting the HOM frequencies for an ideal oblate spheroidal rf cavity. As an example, we applied our analytic treatment to the case of the INDUS-2 cavity [16] which is a part of the synchrotron light source located at the Raja Ramanna Centre for Advanced Technology, Indore [18]. The INDUS- 2 cavity is an elliptically shaped cavity and can be compared with an oblate spheroidal shape. The illustration of the equivalent ellipse drawn on the cavity profile is shown in Fig. 1; it differs slightly from the INDUS- 2 cavity in terms of the geometry, as well as the lack of ports that break the axisymmetry. The axisymmetric $(p=0)$ TM modes of this equivalent cavity profile of INDUS-2 were then determined using the zeros of Eq. (26). For comparison, we also performed detailed three-dimensional FEM electromagnetic simulations of the equivalent INDUS-2 shape, as well as the actual INDUS- 2 cavity (with ports). We also have experimental values of some of the modes (up to $2 \mathrm{GHz}$ ), based on measurements we have made on the INDUS-2 cavity.

Table IV compares the TM mode frequencies obtained from the analytical calculations (column 2), the FEM calculation of the same equivalent oblate shape (column 3), FEM of INDUS-2 cavity full model with all major ports [16] (column 4), and the experimentally measured results of the actual INDUS-2 cavity (column 5). The analytical and FEM calculations for the fundamental mode match very well (within $0.04 \%$ ) whereas the deviation between analytical and FEM full model (including all ports) or experimental values is $\sim 14 \mathrm{MHz}(2.76 \%)$, which is essentially due to the eight port openings in the actual cavity which are not taken into account in the analytical or FEM solution. The analytically obtained higher-order TM mode frequencies show the same trend of excellent agreement with the FEM calculations of the oblate shape and an agreement within 5\% with the full model finite element analysis and with experimental data. The FEM full model agrees very well with experiment, which validates the accuracy of our FEM model, and shows that the difference between the analytical and experimental values is only due to the port openings which are difficult to consider in any analytical approach. The TE axisymmetric modes similarly show a good agreement with FEM and experimental data.

We further applied our semianalytic calculation to a single-cell Tesla cavity (considering midcell dimensions for single cell) [17]. This cavity resonates at $1.3 \mathrm{GHz}$. The equivalent oblate shape for this cavity has $\xi_{o}=0.673$ and $b=0.1154 \mathrm{~m}$. As in the case of the INDUS- 2 cavity, here too, the equivalent oblate shape is close to the actual cavity shape, and the main difference is in the absence of various ports. Table $\mathrm{V}$ shows a comparison of the TM mode frequencies from our calculation and from a 
TABLE IV. TM mode frequency comparison for INDUS-2 rf cavity; analytical results of the axisymmetric oblate shape (column 2), FEM analysis of the same axisymmetric shape (column 3), FEM analysis of cavity shape with all major ports (column 4), and experimental results from INDUS-2 (column 5).

Longitudinal frequency of INDUS-2 rf cavity (GHz)

FEM of INDUS-2

cavity (full model

\begin{tabular}{lcccc}
$\begin{array}{c}\text { TM modes (oblate } \\
\text { shape indices) }\end{array}$ & $\begin{array}{c}\text { Analytic } \\
\text { calculation }\end{array}$ & FEM analysis & $\begin{array}{c}\text { with all major } \\
\text { ports) [16] }\end{array}$ & $\begin{array}{c}\text { Experimental } \\
\text { results }\end{array}$ \\
\hline $1,1,0$ & 0.519 & 0.520 & 0.5058 & 0.5059 \\
$2,1,0$ & 1.049 & 1.048 & 0.9513 & 0.9506 \\
$3,1,0$ & 1.070 & 1.071 & 1.069 & 1.070 \\
$4,1,0$ & 1.505 & 1.504 & 1.432 & 1.430 \\
$3,2,0$ & 1.613 & 1.619 & 1.517 & 1.515 \\
$2,2,0$ & 1.694 & 1.691 & 1.636 & 1.634 \\
$5,1,0$ & 1.989 & 1.989 & 1.961 & 1.923 \\
$4,2,0$ & 2.131 & 2.134 & 2.114 & Not measured \\
$6,1,0$ & 2.160 & 2.160 & 2.127 & Not measured \\
$1,2,0$ & 2.332 & 2.330 & 2.297 & Not measured \\
\hline \hline
\end{tabular}

TABLE V. TM mode frequency comparison for Tesla (ILC) rf cavity (1.3 GHz); analytical results of the axisymmetric oblate shape (column 2), FEM analysis of the same oblate shape (column 3), and FEM analysis of single-cell cavity model with beam aperture opening (column 4).

\begin{tabular}{|c|c|c|c|}
\hline \multirow[b]{2}{*}{$\begin{array}{l}\text { TM modes (oblate } \\
\text { shape indices) }\end{array}$} & \multicolumn{3}{|c|}{ Tesla (ILC) rf cavity frequency $(\mathrm{GHz})$} \\
\hline & $\begin{array}{l}\text { Analytic } \\
\text { calculation }\end{array}$ & $\begin{array}{l}\text { FEM analysis } \\
\text { of equivalent } \\
\text { oblate model }\end{array}$ & $\begin{array}{c}\text { FEM analysis of } \\
\text { single-cell } \\
\text { cavity model }\end{array}$ \\
\hline $1,1,0$ & 1.279 & 1.281 & 1.297 \\
\hline $2,1,0$ & 2.321 & 2.325 & 2.380 \\
\hline $3,1,0$ & 2.651 & 2.650 & 2.687 \\
\hline $4,1,0$ & 3.479 & 3.474 & 3.451 \\
\hline $3,2,0$ & 3.643 & 3.648 & 3.653 \\
\hline $2,2,0$ & 3.970 & 3.976 & 3.940 \\
\hline $5,1,0$ & 4.672 & 4.683 & 4.669 \\
\hline $4,2,0$ & 4.816 & 4.809 & 4.899 \\
\hline $6,1,0$ & 4.925 & 4.932 & 4.999 \\
\hline $1,2,0$ & 5.239 & 5.247 & 5.283 \\
\hline
\end{tabular}

three-dimensional FEM analysis of the equivalent oblate geometry. It can be seen that, again, the agreement is excellent - within $0.15 \%$ for the fundamental, and $0.25 \%$ in the worst case. Further, the comparison with the singlecell cavity model of the ILC (Tesla) cavity, with the beam aperture opening, gives fundamental frequency within $\sim 1 \%$ and HOM to within $2 \%$ in the worst case.

\section{CALCULATION OF THE CHARACTERISTIC PARAMETERS OF AN OBLATE CAVITY}

In the previous section, we have derived a simple curvefitted formula for calculating the TM and TE mode frequencies as a function of the eccentricity (i.e. value of $\left.\xi_{0}\right)$ of the oblate spheroidal shape. Here we extend the treatment to the calculation of the rf properties of an accelerating cavity.

The main parameters required to study characteristic rf cavity modes are the quality factor, shunt impedance, transit time factor and maximum surface electric, and magnetic fields $[19,20]$. These parameters can be determined for each TM and TE mode from the electric and magnetic field distribution for that mode in the rf cavity. For an oblate shape, the electromagnetic field distribution is related to the radial and angular functions which have different spatial behavior for each mode and can be 
calculated using Eqs. (10) and (12). Calculating these functions analytically is very difficult, but it can be done numerically. However, in this manner it is not possible to get field components as a function of $\xi$, $\eta$, etc. Therefore, we have taken the following approach. We evaluate $R$ and $S$ for each $\xi$ and $\eta$ and plot the results. We then fit a polynomial expression (2nd order to 6th order depending on the data) to these plots. This now gives us an analytic expression for the field components, although admittedly the coefficients have to be evaluated numerically for each mode. However, note that these coefficients are now fixed for all $\xi_{0}$, i.e., for all cavity shapes. The methodology used for calculations of the parameters for a typical TM mode is as follows: (1) Evaluate the frequency $\left(f_{m n 0}\right)$ of the mode using geometric parameters such as $a, b$, and $\xi_{0}$, and the values of the coefficients in Eq. (31) from Table II. (2) Calculate $c$ using $c=\pi d f_{m n 0} \sqrt{\mu \varepsilon}$ for that mode. (3) For the particular $c$ value (as calculated above) evaluate the angular function $\left(S_{m n}\right)$ from $\eta=0$ to 1 (in steps of at least 0.05$)$, and the radial function $\left(R_{m n}\right)$ from $\xi=0$ to $\xi_{0}$ (in steps of at least 0.05). (4) Plot the angular and radial functions, and fit polynomial curves in terms of $\eta$ for the angular function and in terms of $\xi$ for the radial function. These fitted curve equations of the angular and radial functions need to be calculated for the particular values of $m$ and $n$. Figures 10 and 11 show the plotted data and the fit for three modes $(m=1, n=1 ; m=2, n=1$, and $m=3, n=1)$, for the radial and angular functions, respectively (for $\xi_{0}=0.5$ ). It can be seen that the polynomial fit is excellent. (5) Substitute these fitted equations for radial and angular functions into Eqs. (23)-(25), to obtain expressions for the magnetic and electric fields. As an example, the expressions for the magnetic and electric fields for the $\mathrm{TM}_{110}$ mode are

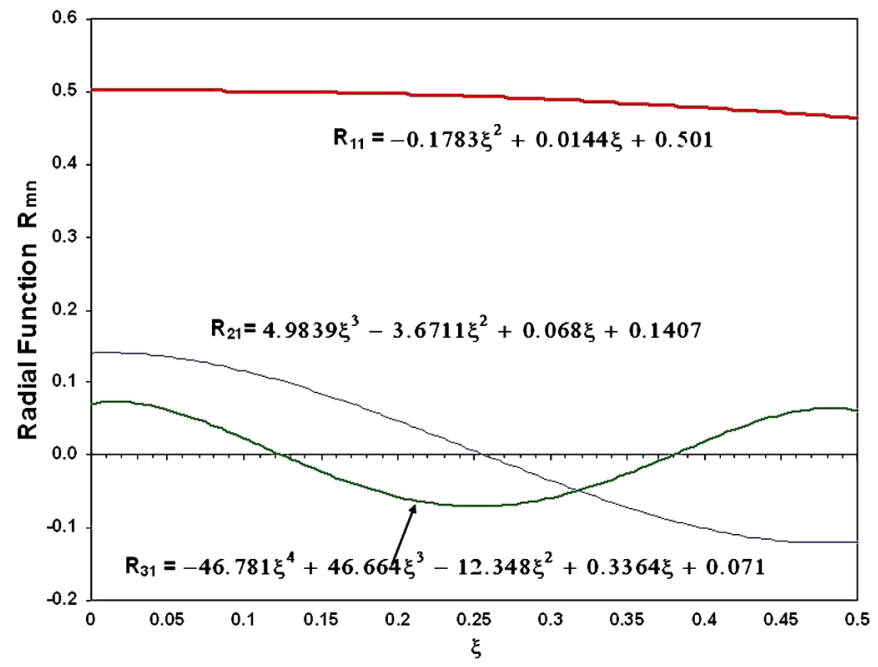

FIG. 10. Oblate spheroidal radial functions and their curvefitting equations for $\xi_{0}=0.5$.

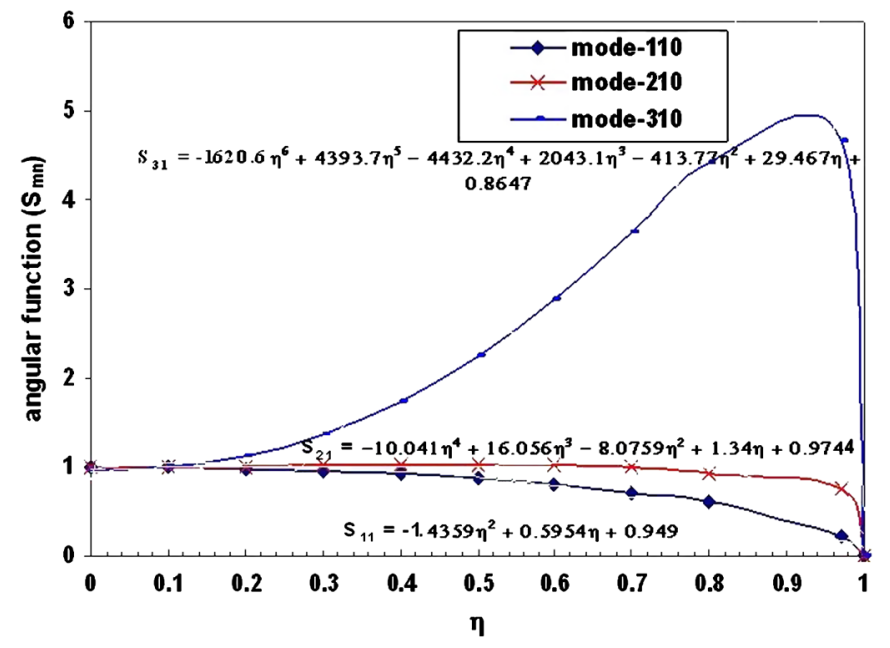

FIG. 11. Oblate spheroidal angular functions and their curvefitting equations.

$$
\begin{aligned}
H_{\phi}= & \frac{A_{n}}{h_{\phi}}\left(-0.1783 \xi^{2}+0.144 \xi+0.5\right) \\
& \times \sqrt{\left(\xi^{2}+1\right)}\left(-1.4359 \eta^{2}+0.5954 \eta+0.949\right) \\
& \times \sqrt{\left(1-\eta^{2}\right)}
\end{aligned}
$$

$$
\begin{aligned}
E_{\xi}= & \frac{A_{n}\left(-0.1783 \xi^{2}+0.144 \xi+0.5\right) \sqrt{\left(\xi^{2}+1\right)}}{j \omega h_{\eta} h_{\phi}} \frac{\partial}{\partial \eta} \\
& \times\left[\left(-1.4359 \eta^{2}+0.5954 \eta+0.949\right) \sqrt{\left(1-\eta^{2}\right)}\right]
\end{aligned}
$$

$$
\begin{aligned}
E_{\eta}= & \frac{A_{n}\left(-1.4359 \eta^{2}+0.5954 \eta+0.949\right) \sqrt{\left(1-\eta^{2}\right)}}{j \omega h_{\xi} h_{\phi}} \\
& \times \frac{\partial}{\partial \xi}\left[\left(-0.1783 \xi^{2}+0.144 \xi+0.5\right) \sqrt{\left(\xi^{2}+1\right)}\right]
\end{aligned}
$$

(6) Now, these field expressions can be used to obtain the characteristic parameters. The constant $A_{n}$ from Eqs. (33)-(35) gets canceled while calculating the characteristic parameters as these parameters are functions of the ratio of the fields. The following three integrals are needed for calculating the different characteristic constants (quality factor, transit time factor, and shunt impedance which are important from the HOM point of view): (a) Voltage gain in cavity gap for any frequency (line integral),

$$
V_{a c c}=2 \int_{0}^{\xi_{0}} a E_{\xi}\left(\xi^{2}+1\right) d \xi
$$

(b) heat dissipated in the cavity (area integral) 
TABLE VI. Comparison of shunt impedance for the INDUS-2 cavity, calculated using our model, and an FEM analysis of the oblate model.

\begin{tabular}{lcc}
\hline \hline TM mode & $\begin{array}{c}R_{\mathrm{SH}} / Q_{0}(\mathrm{Ohms}) \\
\text { analytic model }\end{array}$ & $\begin{array}{c}R_{\mathrm{SH}} / Q_{0}(\mathrm{Ohms}) \\
\text { FEM of oblate shape }\end{array}$ \\
\hline 110 & 40.23 & 46.7 \\
210 & 25.43 & 28.1 \\
310 & 0 & 0 \\
410 & 0.97 & 1.2 \\
\hline \hline
\end{tabular}

$$
P_{d}=\frac{R_{s}}{2} 2 \pi a^{2} \int_{0}^{\eta} \sqrt{\left(\xi^{2}+1\right)}\left(\xi^{2}+\eta^{2}\right) H_{\phi}^{2} d \eta,
$$

where $R_{s}$ is surface resistance; (c) energy stored in the cavity (volume integral)

$$
E_{s}=\frac{\mu}{2} 2 \pi a^{3} \int_{0}^{\xi} \int_{0}^{\eta}\left(\xi^{2}+\eta^{2}\right) H_{\phi}^{2} d \eta d \xi .
$$

From the definition, the quality factor is determined using Eqs. (37) and (38), the transit time factor using the ratio of another line integral of the electric field along the cavity gap having sine or cosine variation (cosine for odd modes and sine for even modes), and Eq. (36). Similarly the shunt impedance can be calculated using Eqs. (36) and (37). The characteristic parameters of the cavity for TE modes can also be evaluated using a similar methodology.

Using the above methodology, the shunt impedance $\left(R_{\mathrm{sh}} / Q_{0}\right)$ for the INDUS-2 oblate shape was calculated, and compared with three-dimensional FEM simulations of the equivalent oblate cavity. This was done for the fundamental mode and three TM HOMs. The data is shown in Table VI. It can be seen that the agreement is quite good for all the modes.

Thus, the semianalytical model we have developed provides a ready method to obtain frequencies and characteristic parameters of $\mathrm{rf}$ cavities within a good approximation by just knowing the ellipse dimensions. For example, the geometric constant $G$ of the Tesla (ILC) cavity for fundamental frequency shows a variation of around $10 \%$ between the analytical (254 $\Omega$ ) and actual finite element model of single cavity (279 $\Omega$ ). The reason for variation in characteristic parameters is due to the aperture openings, for which corrections can be made.

\section{EFFECT OF GEOMETRY VARIATION ON HOM CHARACTERISTICS}

The advantage of the semianalytical formalism we have developed is that we can now systematically investigate the rf properties of cavities as a function of the cavity geometry, and try to understand the effect of geometry on the rf properties of the cavity, and therefore to predict new and better cavity shapes.

\section{A. Effect of geometry variation on HOMs spectrum}

For any oblate shape, the main geometric parameters that affect the electromagnetic properties of the modes are the ellipse major and minor axes $a$ and $b$. Since $\xi$ is evaluated using $a$ and $b$, various combinations of geometric changes in the oblate shape can be worked out using these three values. First, $a$ is kept constant and the $\xi$ is varied in Eq. (31) by varying $b$ which is the accelerating gap of the cavity. The effect of change in $\xi(\Delta \xi)$ on the TM and TE modes is elaborated for constant $a$ in Figs. 12 and 13 , respectively. A change in $\xi$ of around 0.01 changes the frequencies of the TM and TE modes by about $\sim 10 \mathrm{MHz}$ for the INDUS-2 cavity. Further, for changing the cavity fundamental frequency by $1 \mathrm{MHz}$, it is required to change $\xi$ by 0.0013 only. The effect of unit $(1 \mathrm{MHz})$ change in the fundamental frequency on the TM and TE modes is illustrated in Fig. 14. This information can be utilized for the tuning of HOMs.

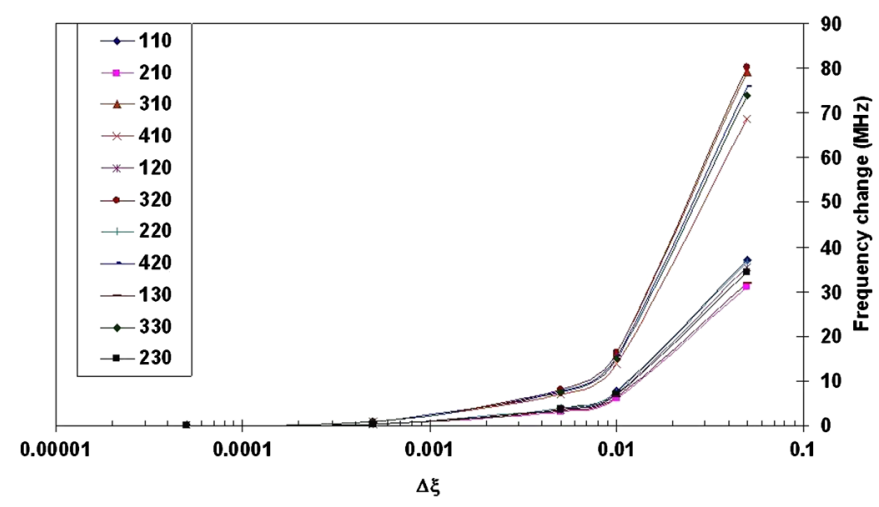

FIG. 12. TM mode frequencies versus change in $\xi$.

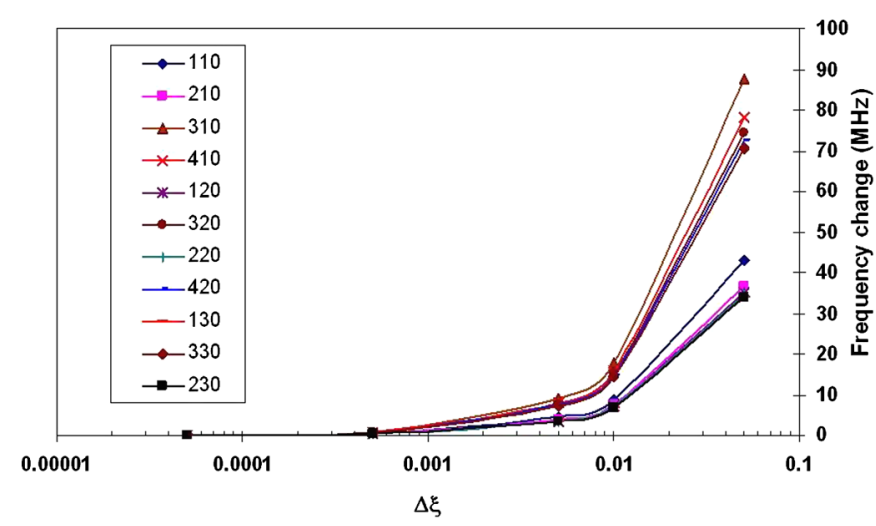

FIG. 13. TE mode frequencies versus change in $\xi$. 


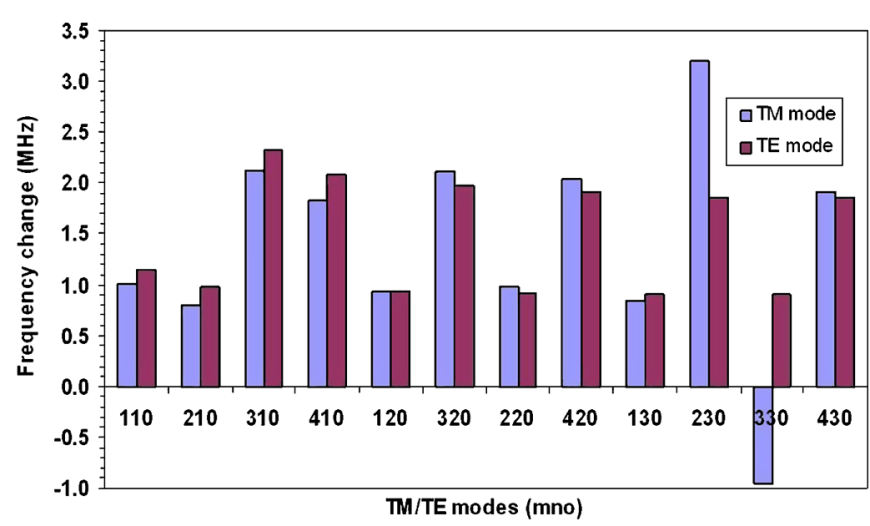

FIG. 14. Effect of changing the fundamental frequency by $1 \mathrm{MHz}$ on the TM and TE HOMs of INDUS-2 equivalent oblate shape cavity.

\section{B. Effect of geometric variation on HOM mode indices}

The effect of increasing $\xi_{0}$ changes the order of the modes as it depends on the geometry of the cavity wall. It is observed that at large $\xi_{0}$ the TM mode indices come in regular ascending order (i.e. $110,120,130$, etc.) whereas at $\xi_{0}=0.1$ the indices are not in such a regular order (i.e. $110,130,120$, etc.). For TM modes the value of $\xi_{0} \geq 0.75$ corresponds to regular ordering of the mode indices. Similar behavior is observed in case of TE modes also; here regular ordering of mode indices occurs for $\xi_{0} \geq 0.8$.

\section{Optimal $\xi$ range for reducing HOMs}

The main characteristic parameters of HOMs are quality factor $\left(Q_{0}\right)$ and shunt impedance. Using the methodology given in Sec. III, these parameters are calculated for oblate shapes for different values of $\xi$. These results are compared with the corresponding parameters for spherical cavities given in [21]. Following are the important observations: (1) The quality factor $\left(Q_{0}\right)$ of the fundamental mode and the HOMs of an rf cavity increases by increasing the $\xi$ of the oblate shape towards that of a sphere. The values of $Q_{0}$ for higher order TM modes of oblate shapes are always more than or equal to the $Q_{0}$ of the fundamental mode unlike in case of a sphere, where the $Q_{0}$ of a few TM modes are less than that of the fundamental mode. (2) The shunt impedance of the fundamental mode increases by increasing $\xi$. As far as the shunt impedance of the TM HOMs is concerned, at higher values of $\xi(\xi>0.8)$, the ratio of the shunt impedance of a few of the HOMs to that of the fundamental mode is $\geq 1$. For lower $\xi$ values $(\xi<0.5)$, this ratio is nearly equal to one for a few of the cases. For a narrow range of moderate $\xi$ values (0.5 to 0.8 ), this shunt impedance ratio has very low values for most of the modes and very few modes have a ratio close to 1 .

To investigate this further, Fig. 15 plots the characteristic parameter ratio $\left(R_{\mathrm{sh}} / Q_{0}\right)_{\mathrm{HOM}} /\left(R_{\mathrm{sh}} / Q_{0}\right)_{f 0}$ of all HOMs up to the $\mathrm{TM}_{420}$ mode (which is generally above the beam

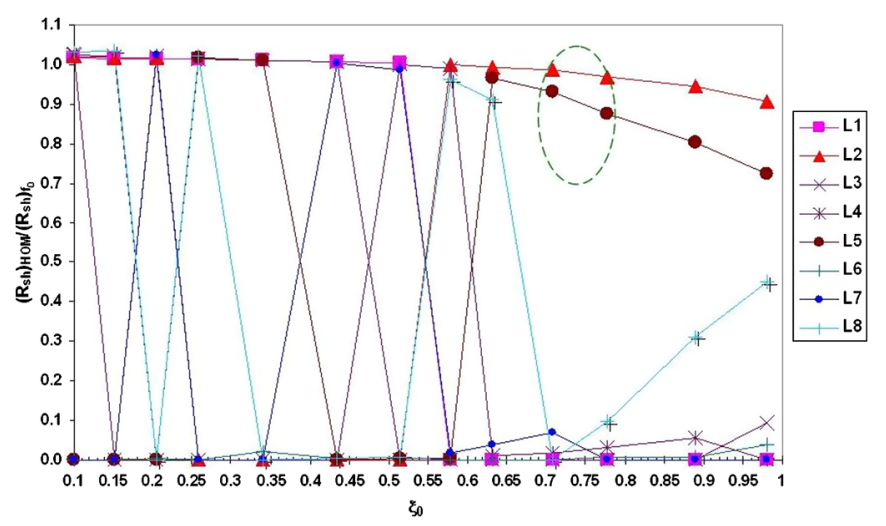

FIG. 15. Effect of $\xi$ on $\left[\left(R_{\mathrm{sh}} / Q_{0}\right)_{\mathrm{HOM}} /\left(R_{\mathrm{sh}} / Q_{0}\right)_{f 0}\right]$ for HOMs.

cutoff frequency), over the range $0.1<\xi<1$. A higher value of this characteristic parameter ratio (above 0.1 ) is considered to represent a significant $\mathrm{HOM}$ which can affect the beam. It is observed from the figure that there are only two significant TM HOMs in the range $\xi=0.69$ to 0.77 . For the ranges $\xi<0.69$ and $\xi>0.77$, there are at least three or more significant TM HOMs. In this range of optimal $\xi$, other parameters such as quality factor, shunt impedance, transit time factor, and peak magnetic and electric field ratios are found suitable as far as the accelerating properties are concerned. Thus, we predict that cavities with $\xi$ in the range $0.69-0.77$ will perform better. This is consistent with the experimental evidence that typical rf cavities are indeed in this range-for example, $\xi=0.673$ for the $1.3 \mathrm{GHz}$ ILC cavity. Of course, these values have been arrived at empirically, over many years of experimental investigations. Our treatment shows that there is a sound theoretical reason for these choices.

\section{SUMMARY AND CONCLUSIONS}

Elliptically shaped $\mathrm{rf}$ cavities have been modeled as oblate spheroids, to obtain the analytical solutions of HOMs and related parameters in order to study the effect of the cavity geometry on HOMs. The present semianalytical study provides a handy tool for calculating the HOM spectrum and its characteristic parameters for an elliptical cavity. The verification of the results with the INDUS-2 and ILC cavity shapes confirms the accuracy of the analytical formulation. This analytical formulation of oblate shapes can now serve as a benchmark for all numerical (FEM/FIT) calculations.

We find that decreasing the $\xi$ value of the oblate cavity decreases the value of TM and TE mode frequencies in the spectrum, hence smaller $\xi$ values have narrow frequency spacing in the spectrum. The behavior of the characteristic parameter ratio shows that, from the point of view of fewer HOMs, a cavity geometry with $\xi$ in the range 0.69 to 0.77 is preferred. This conclusion is consistent with the empirically derived geometries of various operating elliptic cavity shapes, ranging from normal conducting cavities 
in synchrotrons (for example, $\xi=0.51$ for ELETTRA), to superconducting cavities for linacs (for example, $\xi=0.673$ for TESLA and ILC). Our analysis provides a theoretical understanding for why this is the case, and suggests that even higher $\xi$ may be desirable-at least from the HOM point of view.

[1] J.D. Jackson, Classical Electrodynamics (Wiley, New York, 1998), 3rd ed.

[2] S. Belomestnykh and V. Shemelin, in Proceedings of 12th International Workshop on RF Superconductivity (SRF2005), Ithaca, NY, 2005, http://www.lns.cornell.edu/ public/SRF/2006/SRF060424-03/SRF060424-03.pdf.

[3] G. C Kokkorakis and J. A. Roumeliotis, J. Electromagn. Waves Appl. 11, 279 (1997).

[4] G. C. Kokkorakis and J. A. Roumeliotis, J. Electromagn. Waves Appl. 12, 1601 (1998).

[5] L. W. Li, X. K. Kang, and M. S. Leong, Spheroidal Wave Functions in Electromagnetic Theory, Wiley-Interscience Series (Wiley, New York, 2001).

[6] L. W. Li, X. K. Kang, and M.S. Leong, IEEE Trans. Microwave Theory Tech. 51, 922 (2003).

[7] J. A. Stratton, P. M. Morse, L. J. Chu, J. D. C. Little, and F. J. Corbato, Spheroidal wave Functions (Wiley, New York, 1956).
[8] C. Flammer, Spheroidal Wave Functions (Stanford University Press, California, 1957).

[9] M. Abramowitz and I. A. Stegun, Handbook of Mathematical Functions (Dover, New York, 1964).

[10] L. W. Li, M. S. Leong, T. S. Yeo, P. S. Kooi, and K. Y. Tan, Phys. Rev. E 58, 6792 (1998).

[11] W.J. Thompson, Atlas for Computing Mathematical Functions (Wiley, New York, 1997).

[12] W. J. Thompson, Comput. Sci. Eng. 1, 84 (1999).

[13] S. Zhang and J. Jin, Computation of Special Functions, Wiley-Interscience Series (Wiley, New York, 1996).

[14] M. D. Huang and S. Y. Tan, Prog. Electromagn. Res. 82, 241 (2008).

[15] S. Lorenzani and A. Tilgner, J. Fluid Mech. 447, 111 (2001).

[16] V. Jain, U. V. Bhandarkar, S. Yadav, S.C. Joshi, A.D. Ghodke, M. Lad, and P. R. Hannurkar, Nucl. Instrum. Methods Phys. Res., Sect. A 612, 225 (2010).

[17] B. Aune et al., Phys. Rev. ST Accel. Beams 3, 092001 (2000).

[18] D. D. Bhawalkar, G. Singh, and R. V. Nandedkar, Pramana 50, 467 (1998).

[19] E. Jensen, RF Cavity Design (CERN Accelerator School, Daresbury, 2007).

[20] T. Weiland, Nucl. Instrum. Methods Phys. Res., Sect. A 216, 329 (1983).

[21] S. Belomestnykh, Cornell University Technical Report No. SRF 941208-13. 